\title{
Pion-pion correlations in Au+Au collisions at AGS energy
}

\section{Miśkowiec a for the E877 Collaboration ${ }^{b}$}

-Department of Physics, State University of New York at Stony Brook, Stony Brook, NY 11794-3800

bBNL, GSI, INEL, McGill University, University of Pittsburgh, SUNY at Stony Brook, University of São Paulo, Wayne State University

Pion-pion correlation functions have been measured in $\mathrm{Au}+\mathrm{Au}$ collisions at $10.8 \mathrm{GeV} / \mathrm{c}$ per nucleon by the E877 collaboration. The width of the peak in the correlation function for identical pions, which is related to the source size, shows a dependence on pion momenta and on the orientation of the reaction plane.

\section{EXPERIMENT}

Pion-pion correlation functions have been extracted from the Au+Au data taken by E877 in the Fall of 1993. The beam momentum was $10.8 \mathrm{GeV} / \mathrm{c}$ per nucleon and a central trigger ( $10 \%$ of the geometrical cross section) was used. The E877 setup at the AGS consists of three calorimeters and a forward magnetic spectrometer [1,2]. The Target and Participant Calorimeters (TCal and PCal) measure the amount and distribution of transverse energy in the backward and forward hemispheres, respectively, which allows the determination of centrality and reaction plane angle. Pions, kaons, protons and deuterons can be identified in the forward spectrometer, and their momentum is measured with a resolution of $\Delta p / p \approx 2 \%$. Particles of positive and negative charge are measured in one setting. The particle identification plot and the acceptance for pions are shown in Fig. 1.
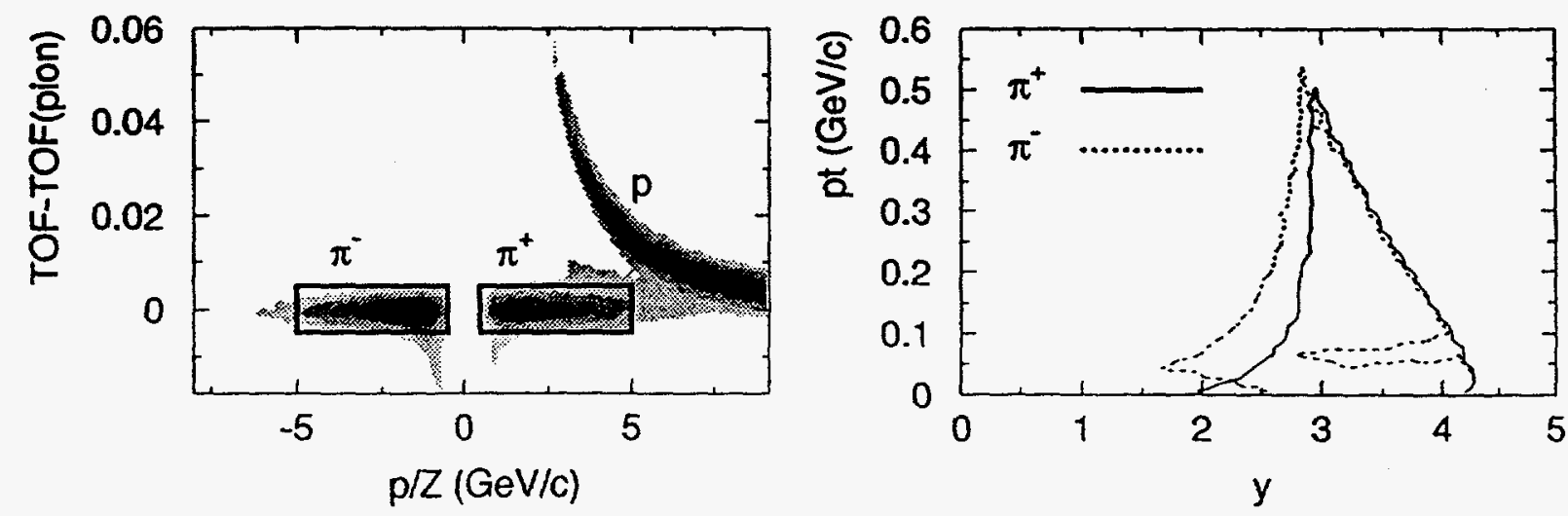

Figure 1. Particle identification (left) and spectrometer acceptance for $\pi^{+}$and $\pi^{-}$for one magnet polarity (right).

\footnotetext{
*Work performed under the auspices of the U.S. Department of Energy. DISTRIBUTION OF TMIS DOCMENT IS UMTMTED 


\section{DATA ANALYSIS}

The number of analyzed $\pi^{+} \pi^{+}, \pi^{-} \pi^{-}$and $\pi^{+} \pi^{-}$pairs was $130 \mathrm{k}, 200 \mathrm{k}$ and $340 \mathrm{k}$. The "doubles-to-singles" correlation functions have been used:

$C\left(Q_{i n v}\right)=\frac{N^{2}}{N(N-1)} \frac{d^{2} N / d p_{1} / d p_{2}}{d N / d p_{1} d N / d p_{2}}$.

The event mixing method was used to determine the denominator of (1). The data were corrected for the tracking efficiency for close tracks (by rejecting all true and mixed pairs with a separation in any of the drift chambers below some minimum value) and Coulomb interactions (by weighting the mixed pairs with a Gamov factor). The variable $Q_{i n v}$, which we used to construct one-dimensional correlation functions, is defined as the momentum difference in the pair c.m. frame. For multi-dimensional correlations we used $q_{x}, q_{y}, q_{x}$, the three components of the momentum difference vector $\vec{q}$ in a coordinate frame moving along the beam $(z)$ axis with half of the beam rapidity. The spectrometer opening in the horizontal $(x)$ direction is about 5 times larger than in vertical $(y)$ making accessible mostly the correlation function in $q_{z}$ and $q_{x}$.

\section{PION SOURCE SIZE}

The corrected $\pi^{+} \pi^{+}, \pi^{-} \pi^{-}$and $\pi^{+} \pi^{-}$correlation functions $C\left(Q_{i n v}\right)$ are shown in Figure 2. We parametrized $C\left(Q_{\text {inv }}\right)$ as

$C\left(Q_{\text {inv }}\right)=1+\lambda \exp \left(-R^{2} Q_{\text {inv }}^{2}\right)$.

In a simple Bose-Einstein model assuming a Gaussian source, which does not take into account source expansion and lifetime, the related source distribution and the r.m.s. radius (as seen from the pair c.m. frame) would be

$\rho(\vec{r})=\exp \left(-\frac{\vec{r}^{2}}{2 R^{2}}\right)$ and $\sqrt{\left\langle r^{2}\right\rangle}=\sqrt{3} R$.

Thus $R$ can be considered as a rough measure of the pion source size. Table 1 shows the comparison of the extracted radius parameter $R$ with that for $\mathrm{Si}+\mathrm{Pb}$ [3].
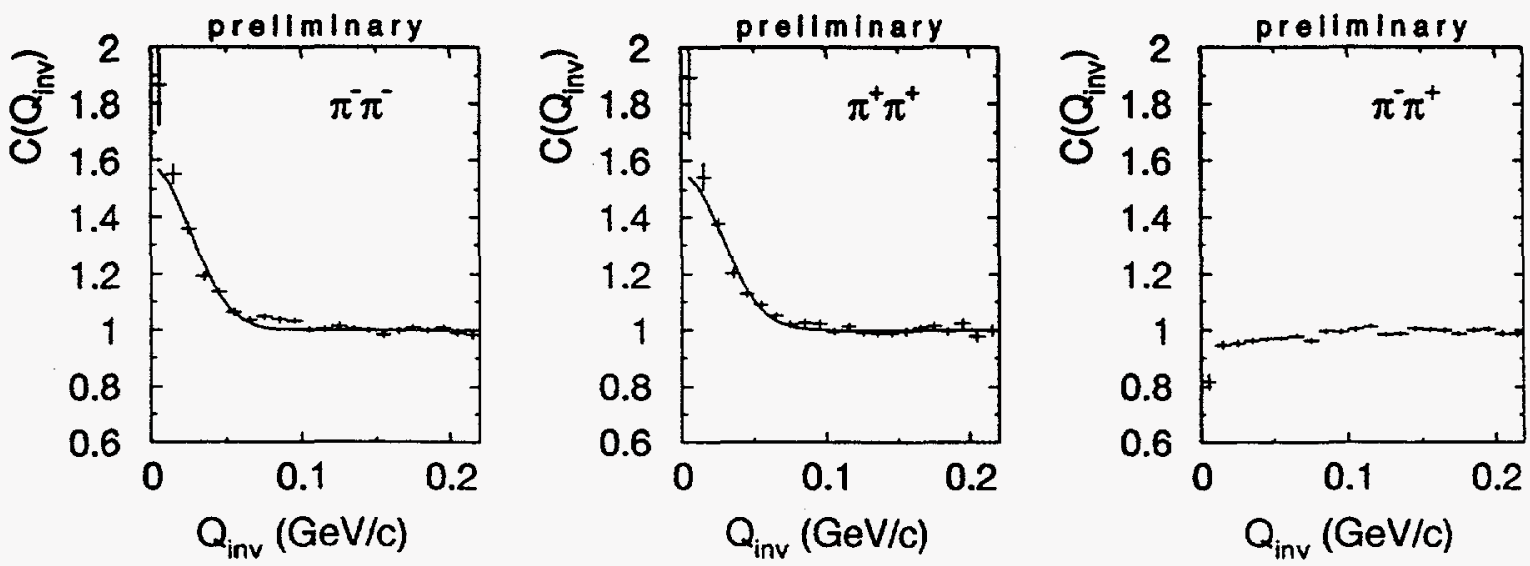

Figure 2. One-dimensional pion-pion correlation functions $C\left(Q_{i n v}\right)$ corrected for double track efficiency and Coulomb interactions. 
Table 1

Radius parameter $R$ for $\mathrm{Au}+\mathrm{Au}$ at $10.8 \mathrm{GeV} / \mathrm{c}$ and $\mathrm{Si}+\mathrm{Pb}$ at $14.6 \mathrm{GeV} / \mathrm{c}$ per nucleon

\begin{tabular}{lccc}
\hline & $\mathrm{Au}+\mathrm{Au}$ & $\mathrm{Si}+\mathrm{Pb}$ & ratio \\
\hline$\pi^{-} \pi^{-}$ & $5.3 \pm 0.3$ & $4.4 \pm 0.6$ & $1.2 \pm 0.2$ \\
$\pi^{+} \pi^{+}$ & $5.1 \pm 0.3$ & $3.4 \pm 1.2$ & $1.5 \pm 0.5$ \\
\hline
\end{tabular}

The ratio between $\mathrm{Au}+\mathrm{Au}$ and $\mathrm{Si}+\mathrm{Pb}$ is about 1.3 which corresponds to a volume ratio of 2.2. This number is close to the ratio of charged particle densities per unit rapidity between the two systems (2.3) which suggests that the freeze-out densities in both reactions are similar.

\section{PAIR MOMENTUM DEPENDENCE OF THE SOURCE SIZE}

We classified pion pairs according to the pair momentum in the lab frame. For each class a correlation function has been constructed and fitted by (2). The extracted radius parameter $R$ decreases for increasing pair momenta (Figure 3, left part). In order to test the reference frame dependence of this effect we also determined $R$ from a similar fit but

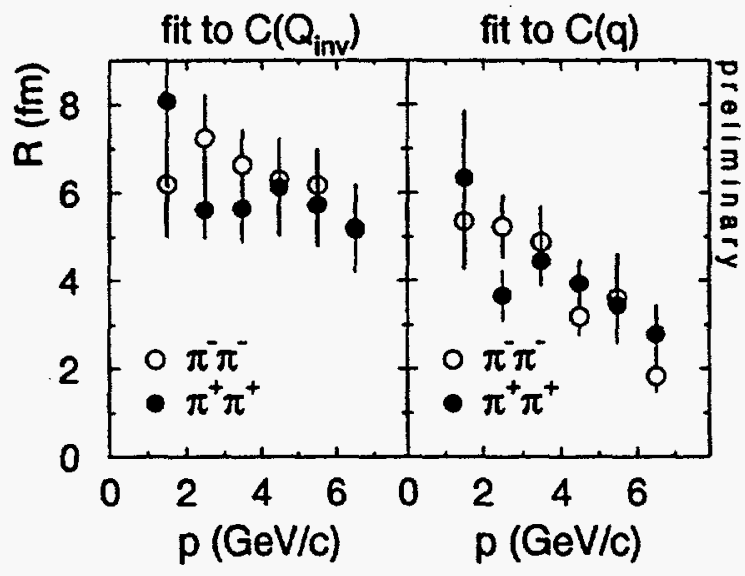

Figure 3. $R$ obtained from the fits of (2) to $C\left(Q_{\text {inv }}\right)$ and to $C(q)$ as a function of pair lab momentum. using $C(q)$ ( $q$ evaluated in the mid-rapidity frame) instead of $C\left(Q_{i n v}\right)$. In this representation the effect is even stronger (Figure 3, right part). This is consistent with the following consideration: The relative momentum is smallest, and thus $R$ biggest, when looking from the pair c.m.s. Consequently, the transformation from the source frame to the pair c.m.s. makes the source look bigger by a factor which increases with the relative velocity between these two frames.

A similar momentum dependence has been observed previously at Bevalac [4] and CERN [5] energies. It may be related to the source expansion [6].

\section{DEPENDENCE ON THE REACTION PLANE ANGLE}

The reaction plane is experimentally defined by the beam axis and the vector $\vec{v}_{1}=$ $\left(v_{1}^{x}, v_{1}^{y}\right)$ which is calculated by adding up unit vectors pointing azimuthally to calorimeter cells with the weight factor equal to the transverse energy deposited in each cell $[2,7]$ :

$v_{1}^{x}=\sum_{i}( \pm 1) E_{T}^{i} \cos \phi_{i} / \sum_{i} E_{T}^{i}$

$v_{1}^{y}=\sum_{i}( \pm 1) E_{T}^{i} \sin \phi_{i} / \sum_{i} E_{T}^{i}$.

The sum goes over all calorimeter cells of PCal with $\eta>1.8(+1)$ and TCal $(-1)$. Thus, $\vec{v}_{1}$ points in the direction in which the most transverse energy is carried by the particles 
emitted with forward rapidities. We divided the events into four classes according to the azimuthal angle of $\vec{v}_{1}: \vec{v}_{1}$ pointing to the left $\left( \pm 45^{\circ}\right)$, to the right, up and down (looking along the beam axis). Since the spectrometer acceptance for pion pairs is located on the right side (we are neglecting pion pairs in a small part of acceptance which is on the left) we will refer to these event classes as opposite, same, perpendicular (up) and perpendicular (down), respectively. For each event class a two dimensional correlation function $C\left(q_{x}, q_{z}\right)$ (average of $\pi^{+} \pi^{+}$and $\left.\pi^{-} \pi^{-}\right)$has been constructed from pairs with $\left|q_{y}\right|<50 \mathrm{MeV} / \mathrm{c}$ and fitted by

$C\left(q_{x}, q_{z}\right)=1+\lambda \exp \left(-R_{x}^{2} q_{x}^{2}-R_{z}^{2} q_{z}^{2}\right)$.

Table 2

Transverse $\left(R_{x}\right)$ and longitudinal $\left(R_{z}\right)$ source size extracted from a fit of (6) to $C\left(q_{x}, q_{z}\right)$ for four orientations of the reaction plane (preliminary).

\begin{tabular}{lcccc}
\hline & same & opposite & perp. (up) & perp. (down) \\
\hline$R_{z}$ & $2.8 \pm 0.3$ & $2.3 \pm 0.2$ & $2.9 \pm 0.3$ & $3.1 \pm 0.3$ \\
$R_{x}$ & $4.8 \pm 0.4$ & $5.8 \pm 0.4$ & $5.5 \pm 0.4$ & $4.9 \pm 0.4$ \\
\hline
\end{tabular}

The obtained $R$ values are shown in Table 2. There appears to be a difference between $R_{x}$ for events with the "same" and "opposite" reaction plane orientations (Figure 4). This difference could be qualitatively explained by the elongation of the source in a direction of the transverse flow and emission of pions from the surface rather than from the volume. The latter produces a momentum-position correlation of pions at freeze-out and can be caused by source expansion and/or pion absorption.

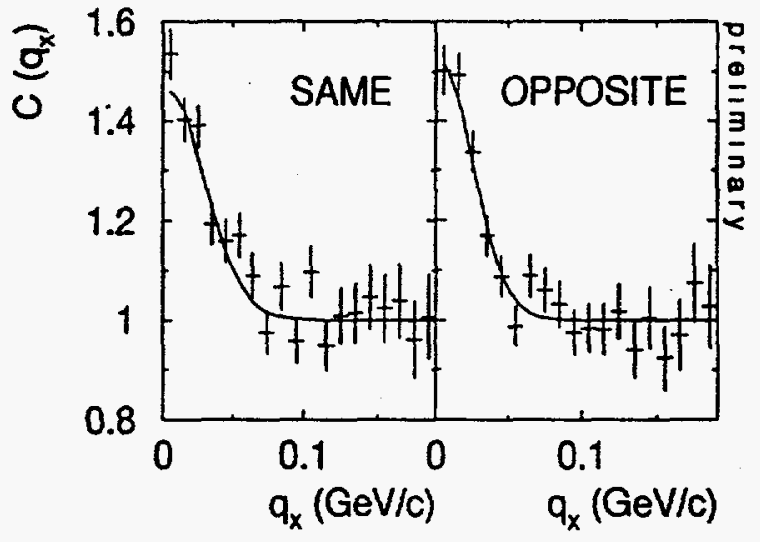

Figure 4. $C\left(q_{x}, q_{z}\right)$ averaged over $0<q_{z}<50 \mathrm{MeV} / \mathrm{c}$ for "same" and "opposite" orientations of the reaction plane. The solid line is the two dimensional fit function averaged over the same range and with the same weighting factor as the data.

\section{REFERENCES}

1. J. Barrette, E877 Collaboration, Phys. Rev. Lett. 70 (1993) 2996.

2. J. Barrette, E877 Collaboration, Phys. Rev. Lett. 73 (1994) 2532.

3. J. Barrette, E877 Collaboration, Phys. Lett. B 333 (1994) 33.

4. D. Beavis et al., Phys. Rev. C 34 (1986) 757.

5. G. Roland, NA35 Collaboration, Nucl. Phys. A566 (1994) 527c.

6. S. Pratt, Phys. Rev. Lett. 53 (1984) 1219.

7. Y. Zhang and J. Wessels, E877 Collaboration, these proceedings. 


\section{DISCLAIMER}

This report was prepared as an account of work sponsored by an agency of the United States Government. Neither the United States Government nor any agency thereof, nor any of their employees, makes any warranty, express or implied, or assumes any legal liability or responsibility for the accuracy, completeness, or usefulness of any information, apparatus, product, or process disclosed, or represents that its use would not infringe privately owned rights. Reference herein to any specific commercial product, process, or service by trade name, trademark, manufacturer, or otherwise does not necessarily constitute or imply its endorsement, recommendation, or favoring by the United States Government or any agency thereof. The views and opinions of authors expressed herein do not necessarily state or reflect those of the United States Government or any agency thereof. 


\section{DISCLAMERR}

Portions of this document may be illegible in electronic image products. Images are produced from the best available original document. 